\title{
Impact of Deprivation on Five Big Personality Dimensions among College Students
}

\author{
Rajendra Revanasiddappa ${ }^{1 *}$, Dr. R, Venkat Reddy ${ }^{2}$
}

\section{ABSTRACT}

India is a developing country in which more than 80 percent of the people are poor and experience deprivation for a prolonged period. This effect of deprivation on individual's personality is very negative. These would hinder the growth \& development of the nation because of the incomplete and partial growth of the individual potentials. The goal of the present study is to impact of the five big personality dimensions of high and low deprivation students. Objectives: To find out the impact of deprivation on five big dimensions of personality among college students. The sample consisted of 600 students; among 300 were high deprived and 300 were low deprived students. Selected students were measured on five big personality scale developed by John and Srivastava (1999). Results indicated that High and low level deprivation students were no significantly differences in the Extroversion, Agreeableness, Consciousness, and Openness to experience of personality dimensions. High and low level deprivation students were significantly differences in Neuroticism of personality dimension.

Keywords: Deprivation and Five Big Personality Dimensions.

India is a developing country in which more than $80 \%$ of the people are poor and experience deprivation for a prolonged period. This effect of deprivation on individual's personality is very negative. These would hinder the growth \& development of the nation because of the incomplete and partial growth of the individual potentials. The youth who have experienced deprivation for a long time would be emotionally insecure and imbalance. The self concept personality factors, adjustment, person perception, time orientation would be on the negative (Melkeri, 1999; Venkat Reddy, 1997; Shobha Salgar, 1996; Venkatesh Reddy, 1992). It is found that sense of deprivation and awareness of deprivation would upset the impact of prolonged deprivation and modify the deprived individual's personality traits adjustment levels emotional intelligence. Motivation syndromes, self concept etc towards positive side (Krishnamurthy and Venkat Reddy, 1995; Venkatesh Reddy, 1994).

\footnotetext{
${ }^{1}$ Assistant Professor, Department of Psychology, Government College Mandya, Mandy -571401, Karnataka, India.

2 Associate Professor, Department of Psychology, Sharanabasaveshwar College of Art, (Deputation to Government First Grade College Afzalpura - 585301

*Corresponding Author

(C) 2015 I R Revanasiddappa, V Reddy; licensee IJIP. This is an Open Access Research distributed under the terms of the Creative Commons Attribution License (http://creativecommons.org/licenses/by/2.0), which permits unrestricted use, distribution, and reproduction in any Medium, provided the original work is properly cited.
} 


\section{Deprivation}

The word deprivation has its origins in the verb "to deprive" which means to dispossess or strip (a person or an object) and it connotes a "felt loss". The reference obviously is to certain deficiencies in the obtaining environment, which are not only there but are also experienced as such by the individual. Therefore, it is argued, when one refers to deprivation the emphasis must be placed on the relevant aspects of the environment which are deficient or wanted in some aspects.

Deprivation in other words means dispossession or taking away of something this taking away can be whole or partial. Used as a construct in psychology it means a state resulting from withdrawal or taking away or withholding supply of a thing to the person and it would become deprivation only when the possession of or availability of it is required for his survival and growth and such an act would endanger his existence either completely or partially. Deprivation as conceived by Reissman (1962) refers to those aspects of middle class culture such as education books formal language from which those (deprived) groups have not been benefited. Wight et al. (1976) have observed that "cultural deprivation must account for deficiencies both in experience and in the conditions of learning".

Langmier (1972) has viewed deprivation as a general phenomenon and articulated the same as insufficient satisfaction of basic needs for a prolonged period. It is also a comprehensive phenomenon which includes sensory, cognitive, emotional and social deprivation. These aspects as is evident overlap one another and in individual cases they are dominant in different ways. Rath (1975) has identified three main features in the term deprived in terms of academic inadequacies in case of deprived child i.e., (i) progressive decline in intellectual functioning (ii) accumulative academic achievement deficit and (iii) premature school termination or higher dropout rate.

\section{Personality}

The word personality is derived from the Latin term persona, which is the mask actor Wore to portray the given characters. The original concept of personality has undergone a tremendous change and it now does not relate to appearance, it goes far beyond and deeper than mere appearance or outward behavior. In one of the definitions Morton Prince (1929) remarked personality is the sum total of all the biological innate dispositions, impulses, tendencies, appetites and instincts of the individual and the dispositions and tendencies by experience. This definition of Morton Prince was criticized on the grounds that it doesn't present any integrated and organized view of personality. Personality cannot be defined through merely summing up the various elements involved in ones personality. Watson (1936) conceived personality as the sum of activities that can be discovered by actual observations over a long enough period of time to give reliable information about the individual behavior. Mc Gill (1949) define "personality as the organization of an individual's needs and potentialities" 
William Stern (1935) observes "personality as a multiform dynamic unity and one ever fully achieves a perfect unity, but always has this as his aim". Raven (1950) says that "personality may refer to several aspects of the human individual as he is himself". Another personologist stresses upon the observable behavior of the individual in personality and states that "personality consists of observable behavior and it is also individual and intrinsic. It is defined as an individual's typical or consistent adjustment to his environment".

The Big-Five factors have become the most prominent model for describing the structure of personality traits (Rammstedt, Goldberg \& Borg, 2010). According to Gosling et al. (2003) The Big Five frameworks are a hierarchical model of personality traits with five broad factors, which represent personality at the broadest level of abstraction. It consisted of neuroticism, extraversion, openness (openness to experience), and conscientiousness and agreeableness variables. Each bipolar factor (Extraversion vs. Introversion) summarizes several more specific Facets (Sociability), which, in turn, subsume a large number of even more specific traits (talkative, outgoing). Alarcon et al. (2009) says that Extraversion refers to individuals' tendency to conversableness, sociability and assertiveness. Agreeableness refers to individuals' tendency to rely on others and concern them. Conscientiousness refers to individuals' diligence, tendency toward planning and organizing, work ethic, self-regulation and assiduousness.

John et a1. (2008) defined the Big Five personality traits as follows: Extraversion is an energetic approach toward the social and material world and includes traits such as sociability, activity, assertiveness, and positive emotionality. Agreeableness contrasts a prosocial and communal orientation toward others with antagonism and includes traits such as altruism, tendermindedness, trust, and modesty. Conscientiousness refers to socially prescribed impulse control that facilitates task and goal directed behaviour, such as thinking before acting, delaying gratification, following norms and rules, and planning, organizing, and prioritizing tasks. Neuroticism contrasts emotional stability and even temperedness with negative emotionality, such as feeling anxious, nervous, sad, and tense. Openness is the breadth, depth, originality, and complexity of an individual's mental and experiential life.

Emotional aspects of extraversion and neuroticism can motivate individuals' behaviours, including behaviours related to deprived (Zellars, Perrewe, \& Hochwarter, 2000). According to Peterson (2000), the optimism frequently exhibited by extraverts "leads to desirable outcomes because it predisposes specific actions that are adaptive in concrete situations". Neuroticism refers to individual's capability to endure such feelings as fear, sin and failure (Alarcon et al., 2009; Costa, \& McCrae, 1992; Bono, \& Judge, 2003). Kim et al conducted a research in hotel industry using the Big-Five Personality model. The results show that extraversion has a negative relationship with job burnout; agreeableness has a negative relationship with pessimism; conscientiousness and agreeableness have a positive relationship with efficiency; and neuroticism has a positive relationship with job exhaustion and pessimism. 
In this study an attempt is made to understand the Impact of Deprivation and deprivation awareness on five big personality dimensions and emotional intelligence of college students. In the back drop of Prime Minister Dr. Manmohan Singh call for inclusive growth of all people this study assumes a very great significance since the findings of this study would help develop intervention strategies and counseling evidence programs and counseling sessions for the development of deprived college students to make them self actualizing and fully functioning persons while removing the mental barriers and behavioral conditioning due to prolonged deprivation.

\section{METHOD}

\section{Objectives}

1. To find out the impact of deprivation on five big dimensions of personality among students.

\section{Hypotheses}

1. There is significant difference in the five big personality dimensions of high and low deprived students.

Participants: The participant group consists of total 600 students, among them 300 high deprived and 300 low deprived students. Participants were selected from degree belonging to Arts, Science and Commerce students of various colleges of Mandya. An equal number of boys and girls were purposively selected from between the ages from 18 to 24 years and the mean age was 21.5 years. The study was conducted during the year January 2013 to June 2013.

The investigator adopted survey method for the collection of information related to personal and socio-demographic status of the respondents. Before the collection of the data the investigator briefed the respondents about the purpose of the study and ensured them that their information would be used only for the research purpose. This process helped to establish rapport with the respondents. To meet the objectives of the present study the data is collected by administering personal information sheet, prolonged deprivation scale, and five big personality dimensions scale was administered to the respondents of different colleges of Mandya.

Personal Information Schedule (PIS): Personal Information Schedule was prepared by investigator. This schedule includes identification data and index variables such as: Name, Age, Gender, Domicile, Education, Type of College (Government or Private College), Socioeconomic status details of the students' sample.

Prolonged Deprivation Scale: To assess the socio-cultural deprivation of the subjects the prolonged deprivation scale developed by Misra and Tripathi (1980) was used. The scale consists of 96 statements with likert type five alternative responses. The five alternative answers are assigned with score values of $1,2,3,4$, and 5 respectively except the statements 70, 74, 75 and 77. For these statements the score values are assigned inversely for the responses A, B, C, D and E. Total score is obtained by adding the scores of all 96 statements. Higher score indicates 
higher level of deprivation. On the basis of the obtained score by the subjects, individuals with a very high score namely above 75 percentile was considered as highly deprived, while low scores namely below 25 percentile was considered as individual with low degree of deprivation.

Five big personality dimensions scale: This section contains a 46 item scale which adapted the big five personality inventory which consists of extroversion, agreeableness, consciousness, neuroticism and openness to experience. Big five inventory (BFI) which was developed by John and Srivastava 1999 which consists of 46 items for five dimensions of personality which respondent will use a 5-point scale ranging from 1 (strongly disagree) to 5 (strongly agree) to indicate the degree to which the item describes them.

Procedure: Students were asked to give their socio-demographic details in the prescribed proforma. They were given appropriate instructions and asked to indicate their responses in the respective sheets given to them. Whenever they had doubt in understanding items, the test administrator clarified their doubts in their local language. Data collection was done in one session and a session lasted for about 60-60 minutes approximately. Then the data was scored and statistically analysed by using descriptive and ANOVA techniques.

\section{RESULT AND DISCUSSIONS}

Table No. 1 show Mean, SD and F values for five big personality dimensions of high and low deprived students.

\begin{tabular}{|c|c|c|c|c|c|c|c|}
\hline $\begin{array}{l}\text { Five big } \\
\text { personality } \\
\text { dimensions }\end{array}$ & $\begin{array}{l}\text { Deprivation } \\
\text { level }\end{array}$ & Mean & SD & $\mathbf{N}$ & df & $\mathbf{F}$ & $\mathbf{p}$ \\
\hline \multirow{2}{*}{ Extroversion } & High & 21.82 & 3.708 & 300 & \multirow{2}{*}{1} & \multirow{2}{*}{3.050} & \multirow{2}{*}{.081} \\
\hline & Low & 22.38 & 4.046 & 300 & & & \\
\hline \multirow{2}{*}{ Agreeableness } & High & 32.45 & 5.359 & 300 & \multirow{2}{*}{1} & \multirow{2}{*}{.648} & \multirow{2}{*}{.421} \\
\hline & Low & 32.08 & 6.077 & 300 & & & \\
\hline \multirow{2}{*}{ Consciousness } & High & 33.13 & 5.594 & 300 & \multirow{2}{*}{1} & \multirow{2}{*}{.194} & \multirow{2}{*}{.660} \\
\hline & Low & 32.93 & 5.726 & 300 & & & \\
\hline \multirow{2}{*}{ Neuroticism } & High & 22.89 & 4.554 & 300 & \multirow{2}{*}{1} & \multirow{2}{*}{6.237} & \multirow{2}{*}{.013} \\
\hline & Low & 23.88 & 5.077 & 300 & & & \\
\hline \multirow{2}{*}{$\begin{array}{ll}\text { Openness } & \text { to } \\
\text { Experience } & \end{array}$} & High & 32.07 & 6.426 & 300 & \multirow{2}{*}{1} & \multirow{2}{*}{.051} & \multirow{2}{*}{.822} \\
\hline & Low & 31.96 & 6.268 & 300 & & & \\
\hline \multirow{2}{*}{ Total } & High & 142.37 & 14.965 & 300 & \multirow{2}{*}{1} & \multirow{2}{*}{.511} & \multirow{2}{*}{.475} \\
\hline & Low & 143.21 & 13.780 & 300 & & & \\
\hline
\end{tabular}

Table N0. 1 show Mean, SD and F values for five big personality dimensions of high and low deprived students. Extroversion (Facet of Gregariousness, Assertiveness, Activity, Excitementseeking, Positive emotion and Warmth) entered into the first personality dimension of high deprived students $($ Mean $=21.82 ; \mathrm{SD}=3.708$ ) and low deprived students (Mean $=22.38$; $\mathrm{SD}=4.046)(\mathrm{F}(1,598)=3.050 ; \mathrm{p}<.081)$ indicating no significant difference. High and low level 
deprivation students were no significant differences in extroversion of personality dimension. Therefore, formulated $\mathbf{H}_{\mathbf{1 . 1}}$, There is significant difference in the five big personality dimensions (extroversion) of high and low deprived students, was not accepted.

Agreeableness (Facet as Trust, Straightforwardness, Altruism, Compliance, Modesty and Tender mindedness) entered into the second personality dimension of high deprived students (Mean = 32.45; $\mathrm{SD}=5.359)$ and low deprived students $($ Mean $=32.08$; $\mathrm{SD}=6.077)(\mathrm{F}(1,598)=.648$; $\mathrm{p}>$.421) indicating no significant difference. High and low level deprived students were no significant differences in Agreeableness of personality dimension. Therefore, formulated $\mathbf{H}_{1.2}$, There is significant difference in the five big personality dimensions (Agreeableness) of high and low deprived students, was not accepted.

Consciousness (Facet as Competence, Order, Dutifulness, Achievement striving, Self-discipline and Deliberation) entered into the third personality dimension of high deprived students (Mean = 33.13; SD = 5.594) and low deprived students $($ Mean = 32.93; $\mathrm{SD}=5.726)(\mathrm{F}(1,598)=.194$; p>.660) indicating no significant difference. High and low level deprivation students were no significant differences in Consciousness of personality dimension. Therefore, formulated $\mathbf{H}_{1.3}$, There is significant difference in the five big personality dimensions (Consciousness) of high and low deprived students, was not accepted.

Neuroticism (Facet as Anxiety, Angry hostility, Depression, Self-consciousness, Impulsiveness and Vulnerability) entered into the fourth personality dimension of high deprived students (Mean $=22.89 ; \mathrm{SD}=4.554)$ and low deprived students $($ Mean = 23.88; $\mathrm{SD}=5.077)(\mathrm{F}(1,598)=6.237$; $\mathrm{p}<.013$ ) indicating highly significant difference. High and low level deprivation students were significant differences in Neuroticism of personality dimension. Therefore, formulated $\mathbf{H}_{\mathbf{1 . 4}}$, There is significant difference in the five big personality dimensions (Neuroticism) of high and low deprived students, was accepted.

Openness to Experience (Faces as Ideas, Fantasy, Aesthetics, Actions, Feeling and Values) entered into the fifth personality dimension of high deprived students (Mean $=32$. 07; $\mathrm{SD}=$ 6.426) and low deprived students $($ Mean $=31.96 ; \mathrm{SD}=6.268)(\mathrm{F}(1,598)=.051 ; \mathrm{p}>.822)$ indicating no significant difference. High and low level deprivation students were no significant differences in Openness to Experience of personality dimension. Therefore, formulated $\mathbf{H}_{1.5}$, There is significant difference in the five big personality dimensions (Openness to Experience) of high and low deprived students, was not accepted.

Overall entered into the five big personality dimensions of high deprived students (Mean = 142.37; $\mathrm{SD}=14.965)$ and low deprived students $($ Mean $=143.21 ; \mathrm{SD}=13.780)(\mathrm{F}(1,598)=$ $.511 ; \mathrm{p}<.475$ ) indicating no significant difference. High and low level deprivation students were no significantly differences in the five big personality dimensions. Therefore, formulated $\mathbf{H}_{\mathbf{1}}$, 
There is significant difference in the five big personality dimensions of high and low deprived students, was not accepted.

The study results reveal that High and low level deprivation students were no significantly differences in the five (exclude neuroticism) big personality dimensions. Zellars, Perrewe \& Hochwarter (2000) it was found that higher and lower deprivation students were no significantly difference in the personality dimensions. The similar results of Alarcon et al. (2009), Bono \& Judge (2003), Peterson (2000), Chang, Rand \& Strunk (2000), Watson, David \& Suls (1999), Piedmont (1993), Costa \& McCrae (1992), Bolger \& Schilling (1991).

Findings of the present study also, indicating that high and low level of deprivation students were no significantly differences in the Extroversion, Agreeableness, Consciousness, and Openness to experience of personality dimensions. It may be positive behavioural of Gregariousness, Assertiveness, Activity, Excitement-seeking, Positive emotion, Warmth Straightforwardness, Altruism, Compliance, Modesty Competence, Order, Dutifulness, Achievement striving, Selfdiscipline, Deliberation Fantasy, Aesthetics, Actions, Feeling and Values of high and low deprived students have positive behavioural access to modern health facilities and awareness of deprivation. Consequently students have may be developing positive personality dimensions.

\section{CONCLUSIONS}

1. High and low level deprivation students were no significantly differences in the Extroversion, Agreeableness, Consciousness, and Openness to experience of personality dimensions.

2. High and low level deprivation students were significantly differences in Neuroticism of personality dimension.

\section{REFERENCES}

Alarcon, G., Eschleman, K. J. \& Bowling, N. A. (2009). Relationship between Personality Variables and Burnout: A Meta-Analysis. Work \& Stress,

Bono, J. E. \& Judge, T. A. (2003). Core self evaluations: A review of the trait and its role in job satisfaction and job performance. European Journal of Personality, 17, 5-18.

Costa, P. T. Jr. \& Mc Crae, R. R. (1992). Normal personality assessment in clinical practice:

Gosling, S. D., Rentfrow, P. J., \& Swann, W. B. Jr. (2003). A very brief Measure of the Big-Five personality domains. Journal of Research in Personality, 37, 504-528.

John, O. P., Naumann, L. P., \& Soto, C. J. (2008). Paradigm shift to the integrative Big Five trait taxonomy: History, measurement, and conceptual issues. In O. P. John, R. W. Robins, \& L. A. Pervin (Eds.), Handbook of personality: Theory and research (pp. 114-158). New York: Guilford Press. 
Krishnamurth, B. and Venkat Reddy, R. (1995). Psychology for India: A socio-psychological theory of oppression. Journal of community Guidance and research, Vol 12(2), 125-134.

Melkeri, S. P. (1999). Person perception in the context of socio-cultural deprivation. Published Ph. D. Thesis, University of Gulbarga, Gulbarga.

Misra, G. and Tripathi, L. B. (1980). Psychological consequences of prolonged deprivation, Agra: National psychological Corporation.

Peterson, C. (2000). The future of optimism. American Psychologist, 55, 44-55.

Rammesdt, B., Goldberg, L. R., \& Borg, I. (2010). The measurement equivalence of Big -Five factor markers for persons with different levels of education. Journal of Research in Personality, 44, 53-61.

Venkat Reddy (1997). The impact of deprivation and deprivation awareness on personality factors, Published Ph. D. Thesis, University of Gulbarga, Gulbarga.

Venkatesh Reddy, A. N. (1992). Impact of deprivation on some dimensions of self. Published Ph. D. Thesis, University of Gulbarga, Gulbarga.

Wight et al. (1976). Culture deprivation: Operational definition in terms of language development. American Journal of Orthopsychiatry, 40, 77-86.

Zellars, K. L., Perrewe, P. L., \& Hochwarter, W. A. (2000). Burnout in health care: the role of the five factors of personality. Journal of Applied Social Psychology 30, 1570-1598. 